\title{
Modulation of sensitization processes in the management of pain and the importance of descending pathways: a role for tapentadol?
}

Filippo Caraci, Flaminia Coluzzi, Franco Marinangeli, Sebastiano Mercadante, Giuseppe Rinonapoli, Patrizia Romualdi, Mariaflavia Nicora \& Anthony H Dickenson

To cite this article: Filippo Caraci, Flaminia Coluzzi, Franco Marinangeli, Sebastiano Mercadante, Giuseppe Rinonapoli, Patrizia Romualdi, Mariaflavia Nicora \& Anthony H Dickenson (2019): Modulation of sensitization processes in the management of pain and the importance of descending pathways: a role for tapentadol?, Current Medical Research and Opinion, DOI: 10.1080/03007995.2019.1703664

To link to this article: https://doi.org/10.1080/03007995.2019.1703664

Accepted author version posted online: 11 Dec 2019.

Submit your article to this journal $\sqsubset$

Цll Article views: 15

Q View related articles $\sqsubset$

View Crossmark data \lceil 
Modulation of sensitization processes in the management of pain and the importance of descending pathways: a role for tapentadol?

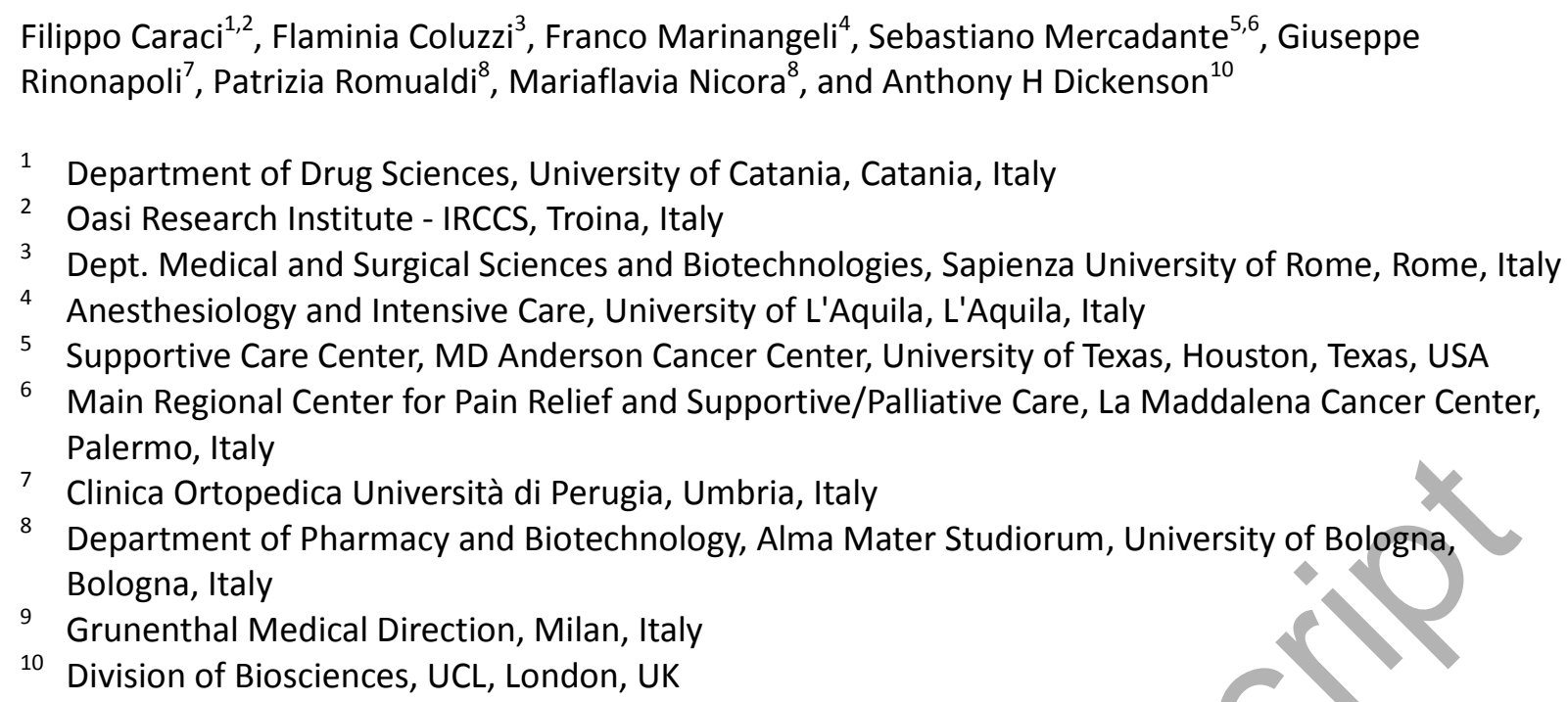

Correspondence: Anthony H Dickenson, Division of Biosciences, UCL, London, UK, email: anthony.dickenson@ucl.ac.uk

\section{Transparency}

\section{Declaration of funding}

Medical writing assistance was supported by Grunenthal.

\section{Declaration of financial/other relationships}

AHD has received speaker fees from Grunenthal, Teva, and Allergan. FCaraci has received speaker fees from Grunenthal, Lundbeck, and Janssen. FColuzzi served as a speaker for Grunenthal, Angelini, and Malesci. PR has received speaker fees from Grunenthal, Angelini, and Molteni. FM has received speaker fees from Grunenthal, MSD, Molteni, and Kyowa Kirin. SM has received speaker fees from Grunenthal, Molteni, Angelini, and Kyowa Kirin. GR has received speaker fees from Grunenthal. FN is an employee of Grunenthal. Peer reviewers on this manuscript have received an honorarium from CMRO for their review work but have no other relevant financial relationships to disclose.

\section{Author contributions}

All authors contributed to the definition of the scopes of this manuscript and the collection of data. AHD wrote the first draft, which was revised by all other authors. All authors have read and approved the final draft.

\section{Acknowledgements}

Editorial assistance was provided by Luca Giacomelli, $\mathrm{PhD}$, whose assistance was supported by Grunenthal, and Aashni Shah and Sara Di Nunzio (Polistudium srl, Milan, Italy), whose assistance was supported by internal funds. 


\section{Abstract \\ Objective}

This paper presents and discusses recent evidence on the pathophysiological mechanisms of pain. The role of tapentadol-an analgesic molecule characterized by an innovative mechanism of action (i.e., $\mu$-opioid receptor [MOR] agonism and inhibition of noradrenaline [NA] reuptake [NRI]) -in the modulation of pain, and the most recent pharmacological evidence on this molecule (e.g., the $\mu$-load concept) are also presented and commented upon.

\section{Methods}

Narrative review.

\section{Results}

Solid evidence has highlighted the importance of central sensitization in the transition from acute to chronic pain. In particular, the noradrenergic system holds a major role in limiting central sensitization and the progression to chronic pain. Therefore, pharmacological modulation of the noradrenergic system appears to be a well-grounded strategy for the control of chronic pain. Tapentadol is characterized by a to-date-unique mechanism of action, since it acts both as a MOR agonist and as an inhibitor of NA reuptake. The synergistic interaction of these two mechanisms allows a strong analgesic effect by acting on both ascending and descending pathways. Of note, the reduced $\mu$-load of tapentadol has two important consequences: first, it limits the risk of opioidrelated adverse events, as well as the risk of dependence; second, the NA component becomes predominant at least in some types of pain with consequent specific clinical efficacy in the treatment of neuropathic and chronic pain.

\section{Conclusions}

According to these characteristics, tapentadol appears suitable in the treatment of chronic pain conditions characterized by both a nociceptive and a neuropathic component, such as osteoarthritis or back pain.

Keywords: tapentadol; neuropathic pain; pharmacology; $\mu$-load 


\section{Introduction}

Pain is an unpleasant sensory and emotional experience associated with actual or potential tissue damage or described in terms of such damage with a major impact on quality of life (QoL) [1]. Within the overall pain experience, the sensory components related to the transmission of the pain stimulus from the peripheral site of injury to the central areas of the brain via the spinal cord (ascending pain pathway) and the physiological mechanisms of modulation (descending pain pathway) converge with psychological, internal and affective components, which together generate the individual pain experience [1]. Therefore, pain represents an individual and subjective experience, with consequent difficulties in defining both methods of assessment and optimal therapies.

Research has therefore centered on a deeper investigation of the molecular mechanisms which lead to the experience of pain, with the aim of identifying new treatments targeting the specific pathophysiological mechanisms that promote establishment of chronic pain.

This paper will present and critically discuss the most recent pharmacological evidence on tapentadol [2], an analgesic molecule characterized by an innovative mechanism of action - i.e., $\mu$-opioid receptor (MOR) agonism and inhibition of noradrenaline (NA) reuptake (NRI) [3] - and its role in the modulation of pain, on the light of the most recent evidence on the pathophysiological mechanisms of pain, leading to the establishment of chronic pain. Finally,

\section{Pathophysiology of pain: basic knowledge Current definitions}

Pain can be defined according to: (i) the nature and location of the stimulus; (ii) etiology; and (iii) duration and therapeutic response (Table 1) [1]. Remarkably, in distinguishing between acute and chronic pain, the mechanisms can be more relevant than simply the duration of pain [1]. Indeed, peripheral damage can lead to enhanced excitability of peripheral nociceptors, with consequent peripheral sensitization and primary hyperalgesia. This causes a constant stimulation of the central nociceptive transmission pathways, leading to secondary hyperalgesia and central sensitization through spinal changes (neuroplasticity) [4].

\section{Pain pathways}

Pain pathways are a complex sensory system able to detect and integrate a protective response both conscious and subconscious - to noxious stimuli by transducing them and projecting to CNS by primary afferent, thinly myelinated or unmyelinated nerve fibers (A-delta and C-fibers, respectively) (Figure 1) $[5,6]$.

The simple transmission of pain signals from the spinal cord onwards fails to account for the complex interactions occurring in the dorsal horn and elsewhere as the pain becomes persistent. Central sensitization of dorsal horn neurons represents a form of long-term plasticity in the CNS, and often occurs following nerve injury and inflammation. Central sensitization is characterized by increased excitability of dorsal horn neurons, increased spontaneous activity, wider receptive areas, and enhanced responses evoked by primary afferent fibers [7]. The mechanism underlying central sensitization involves glutamate released by $\mathrm{C}$-fibers onto post-synaptic $\mathrm{N}$-methyl-D-aspartate (NMDA) receptors, which cause calcium influx. Indeed, central sensitization involves changes in deep spinal cord neurons, which mimic some aspects of the supraspinal plasticity that characterizes learning and memory [7].

Supraspinal involvement is central to the experiential aspects as well as in the affective components of pain. Multiple regions are involved, such as the hypothalamus, amygdala and cortex, as well as the nucleus accumbens and the periaqueductal grey $[5,8]$. Pathways run back from the brain to the spinal cord and many of the descending tracts are monoaminergic: at spinal synapses, the 
descending pathways induce the release of several mediators, including endogenous opioids, NA, serotonin (5-HT) and gamma-aminobutyric acid (GABA), thus modulating the transmission between primary and secondary neurons [9]. Remarkably, NA pathways exert an inhibitory effect on the transmission of acute and chronic pain, thus counteracting the establishment of chronic pain [9]. Conversely, serotoninergic pathways may have a facilitatory effect in the advanced stages of chronicity and therefore, might play a pro-nociceptive role [10]. Imbalance between amplified spinal ascending signals and inadequate activation of the descending inhibitory pathways plays a key role in the development and maintenance of many chronic pain syndromes [9]. Activity in the descending noradrenergic system can be gauged in animals and humans by diffuse noxious inhibitory controls (DNIC) and conditioned pain modulation (CPM), respectively, whereby one painful stimulus can inhibit another [11]. This control mechanism often fails in persistent pain states, allowing descending facilitations from the rostral ventromedial medulla (RVM) to dominate.

Glial cells also play a role in the release of neuromediators and other molecules involved in pain development and processing (e.g., calcitonin gene-related peptide, substance $P$ and glutamate) [12]. In addition, glia can release pro-inflammatory cytokines [5]. The multifaceted interaction between glia and neurons depends on several factors, including glial cell types, location of the regulatory process (peripheral nerve, spinal cord or brain) and type of the pain. In particular, NA has a protective effect on pro-inflammatory glia activation [13].

According to this evidence, it has been proposed that the rescue of the noradrenergic system has a major clinical relevance in preventing the transition from acute to chronic pain [13].

\section{Clinical relevance}

The functional and structural modifications of the nervous system in response to pain are likely to have immediate clinical relevance [14]. As discussed, establishing a diagnosis of chronic pain in a patient with active neuroplasticity phenomena should not rely on the duration of symptoms alone. Indeed, functional and structural abnormalities of the nervous system and their clinical relevance has been documented in some conditions, including osteoarthritis (OA) and chronic back pain (CBP) [15].

Depending on the nature and location of the stimulus and the originating mechanisms, the pain is distinguished as somatic, visceral or mixed pain [1]. In turn, considering its etiology, pain can be defined as nociceptive (nociceptor activation by high threshold stimuli and their transmission to the central nervous system), neuropathic (induced by central or peripheral nervous system diseases or lesions), nociplastic where pain is present without an obvious peripheral generator and mixed (including the previous components) [1].

Several diagnostic tests (response to heat, mechanical allodynia, punctate hyperalgesia and temporal summation) are available in clinical practice to detect central sensitization [16].

\section{Osteoarthritis}

Patients with severe OA can show some sensory abnormalities, such as loss of cutaneous vibration sensitivity, hypoesthesia to mechanical, pinprick and thermal stimuli and mechanical allodynia [17]. In addition, pain does not match the innervation territories of peripheral nerves or nerve roots.

Animal models of OA have shown the involvement of ectopic sprouting of sensory and sympathetic nerve fibers in the painful arthritic joint [18-20]. Moreover, blocking the actions of spinal $\alpha-2-$ adrenergic receptors completely abolishes DNIC, thus suggesting the key role of the noradrenergic system in DNIC $[11,20]$. Furthermore, as a tonic descending noradrenergic tone is always crucial for the expression of DNIC, variations in descending serotonergic signaling over the course of the model mean that the noradrenergic component plays a more relevant role in states of sensitization. Loss of this control allows descending facilitation to gain in function. 
Referred pain in OA patients is thought to be mediated by central sensitization following continuous nociceptor firing in and around the affected joint $[21,22]$. As a further confirmation of pain sensitization in OA patients, a pooled analysis of 15 studies examining pressure pain thresholds (PPTs) and heat pain thresholds disclosed large differences in PPTs between knee OA patients and controls and moderate PPT differences between knee OA participants with high symptom severity compared with those with low symptom severity [23]. In a cross-sectional study on 53 people with knee OA scheduled to undergo primary total knee arthroplasty [24], pain frequency maps revealed enlarged areas of pain, especially in women, associated with higher knee pain severity and stiffness, lower pressure pain thresholds at the knee and epicondyle, and higher scores on the central sensitization inventor, thus further supporting the notion of chronic sensitization in patients with knee OA.

In a very recent clinical study on 282 patients with hand OA [25], peripheral and central sensitization both emerged as independent contributors to pain, regardless of psychological factors and radiographic severity. Sensitization may therefore represent a possible treatment target. In another recent analysis, Carlesso et al. evaluated 852 individuals free of persistent knee pain from the Multicenter Osteoarthritis Study, a longitudinal cohort of older adults with or at risk of knee OA [26]. Four pain susceptibility phenotypes were identified, characterized by varying proportions (low/absent, moderate or high) of pressure pain sensitivity and of facilitated temporal summation, reflecting different measures of sensitization. Subjects in the pain susceptibility phenotypes with a higher-pressure pain sensitivity and a moderate proportion of facilitated temporal summation were twice as likely to develop incident persistent knee pain over 2 years. A recent study used neuroimaging, sensory testing and the Pain DETECT questionnaire to study if central and potential neuropathic changes related to the long-term outcome of replacement surgery [27]. Patients who scored positive on Pain DETECT had greater pain to mechanical and cold stimuli and activity was significantly lower in the rostral anterior cingulate cortex and higher in the RVM, suggesting an enhancement of descending facilitation. Neuropathic-like pain before surgery and higher neural activity in the RVM were linked with moderate-to-severe long-term pain after arthroplasty. This clear demonstration of reductions in inhibition and increases in facilitation of nociceptive signaling with an impact on worse outcome after arthroplasty would argue for attempts to restore normal descending modulation in these patients before surgery, which could reduce persistent pain after surgery.

On these bases, prevention or amelioration of central sensitization can represent a novel pharmacological approach to preventing onset of chronic pain in OA [28].

\section{Chronic back pain}

Central sensitization leading to chronic pain also plays a major role in CBP [15]. Apkarian et al. compared the brain morphology of 26 CBP patients to that of matched controls and showed that CBP patients presented 5-11\% less neocortical grey matter volume than controls (the equivalent of grey matter volume lost in 10-20 years of normal aging) [29]. The amount of decreased volume was also increased with pain duration, with a $1.3 \mathrm{~cm}^{3}$ loss of grey matter for every year of chronic pain. These results suggest that $\mathrm{CBP}$ is associated with neurodegeneration and brain atrophy. In a more recent study, Fritz et al. investigated the association of CBP and regional grey matter volume, confirming that $\mathrm{CBP}$ is associated with decreased regional grey matter in several areas of the brain [30]. Noteworthy, treating chronic pain might contribute to restore normal brain function, as suggested in a pilot study by Seminowicz et al. [31].

Effective involvement of inhibitory descending pathways could protect against the development of chronic pain [27]. Moreover, the activation of the opioid receptor is particularly relevant in acute nociceptive pain, while the inhibition of NA reuptake activity plays a crucial role in chronic neuropathic pain [9]. 


\section{The selection of appropriate therapy}

The prevention and management of chronic pain and central sensitization should target the specific mechanisms involved for each single patient $[15,32]$ but at present, individualized, tailored, mechanism-based therapy is currently not possible in the large majority of cases. Two basic criteria should be met when selecting the analgesic strategy: therapeutic appropriateness and timing. In particular, the selected drug must target the main mechanisms responsible for the particular type of chronic pain, while tackling central sensitization and controlling the nociceptive and neuropathic components.

Basically, two methods of damping central sensitization are known, namely blocking the peripheral drive, which sustains the sensitization and interacting with the central transmitter systems involved in its generation and modulation. Although it has been suggested that both approaches may generally have short-lasting effects and may be challenging to administer [32] it is possible to rescue descending inhibitory controls with drugs that elevate NA levels and potentiate noradrenergic system. In turn, this increased inhibitory tone will modulate both central sensitization and offset descending facilitations, both of which will promote pain.

Moreover, a multimodal and multi-mechanistic approach to pain management must be considered in clinical practice [15]. For instance, in the case of OA, treatment of pain should, in an ideal world, pursue three goals: inhibit peripheral mechanisms of pain and inflammation, treat central pain mechanisms and reduce progression of joint degeneration (peripheral pain generators) [33]. While these goals were described for $\mathrm{OA}$, they may also be applied to other conditions, with the aim to restore normal modulation of the painful mechanisms [14,32].

Therefore, acute pain associated with an inflammatory phenomenon (e.g., post-traumatic inflammation), minor trauma or transient musculoskeletal pain can be treated by non-steroid antiinflammatory drugs (NSAIDs) and paracetamol. When pain exhibits signs of progression (enlarged susceptible areas, secondary allodynia), the use of NSAIDs becomes no longer sufficient since spinal sensitization phenomena due to underlying functional plasticity are modifying the course of the pain disease. Furthermore, NSAIDs are not recommended for prolonged treatment because of the risk of side effects and are therefore not suitable for treating neuropathic pain [14].

Once the chronic stage has been reached, therapeutic intervention may no longer fully reverse the plastic alterations in the nervous system. Modulation of the noradrenergic pathway by appropriate treatments may, in principle, modulate neuropathic pain [34]. The descending noradrenergic tone slows the appearance of allodynia and hyperalgesia [35]. In addition, nerve injury downregulates MOR expression [36].

Evidence demonstrating the relevance of the descending noradrenergic pathways in the establishment of chronic pain suggests the use of drugs with a targeted effect on NA reuptake to restore the balance in the descending pathways. Drugs, such as tapentadol or antidepressants, may act in this line by strengthening synaptic inhibition systems in the spinal cord and brain by preventing or eliminating some of the conditions resulting in the maladaptive plasticity of the synapses [14].

\section{The role of tapentadol in modulation of pain}

Tapentadol is a centrally acting analgesic drug, considered the first representative of the MOR-NRI class of drugs. It displays an analgesic efficacy comparable to that of strong classical opioids $[9,37,38]$. The pharmacology of tapentadol has recently been reviewed [9]. Noteworthy, this molecule was synthetized by a rational drug-design program, with the aim of synthetizing a molecule endowed by both MOR and NRI activity with minimal serotonergic actions [38]. Given this peculiar 
mechanism of action, tapentadol can act on central sensitization process, which are also due to the impairment of the descending NA pathways, in a different way if compared with pure opioids which lack the NRI component action.

Despite a 50 -fold lower affinity for MOR than morphine and a relatively moderate NRI activity, the potency of tapentadol is comparable to that of morphine [38]. Such potency cannot be explained by a simple additive effect, but rather by a synergistic interaction between the two distinct mechanisms of action. Therefore, relatively moderate receptor activities are sufficient to achieve a strong analgesic effect by acting on both ascending and descending pathways [9].

With regards to pharmacokinetics, tapentadol follows a linear model with rapid oral absorption $\left(\mathrm{C}_{\max }\right.$ $<2$ hours and $t_{1 / 2}$ of approximately 4 hours) [9]. Since tapentadol is not a prodrug, pharmacokinetics and analgesic efficacy are independent of metabolic activation and individual variations. Moreover, given its glucuronidation-based metabolism, tapentadol is associated with a low risk of drug-drug interactions at the cytochrome (CYP) 450 level [9]. For the same reason, tapentadol and its metabolites are almost completely eliminated by urinary excretion, and tapentadol should therefore be administered with caution in patients with severely impaired renal function.

\section{Pharmacology studies}

In knockout mice with a deletion of MOR, tapentadol partially retained its analgesic efficacy [39], thus confirming the relevance of its NA component in inducing pain relief. Moreover, systemic tapentadol dose-dependently reduced evoked responses of spinal dorsal horn neurons to a wide range of peripheral stimuli in neuropathic animals [40]. In this study, with a wind-up approach, this direct measure of events behind central sensitization was reduced by the drug with greater effect on neuropathic pain than controls. Moreover, application of opioid receptor or selective $\alpha-2-$ adrenoceptor antagonists produced near complete reversal of the inhibitory effects of tapentadol, thus suggesting that only MOR-NRI is involved in analgesia. The NA component appeared more important in the neuropathic animals. In another animal study, the effects of systemic morphine, tapentadol or duloxetine were evaluated in rats with spinal nerve ligation (SNL) or sham surgery [39]. All molecules produced reversal of tactile hypersensitivity in SNL animals, but only tapentadol induced a significant increase in spinal NA levels in SNLs, thus offering a mechanistic correlate to the clear differentiation between a pure MOR and MOR-NRI. In another study in a rat model, tapentadol inhibited the spontaneous electrophysiological activity of locus coeruleus in a dose-dependent fashion [41]. This inhibitory effect was reversed by both $\alpha$-2-adrenoceptor antagonists and MOR antagonists. In a model of cancer pain, tapentadol administration inhibited evoked spinal neuronal activity with efficacy against mechanical, thermal and electrically evoked activity [42]. As shown in previous studies, the effects of tapentadol were fully reversible by the MOR antagonist naloxone and partially by the $\alpha$-2-adrenoceptor antagonist atipamezole, again supporting the idea of MOR-NRI synergistic actions. Last, tapentadol seems to selectively protect from splenic cytokines, differing from morphine, which exerts a generalized suppression on all cytokines [43].

Recently, Lockwood and Dickenson explored a combination therapy of the centrally acting analgesic agents tapentadol and pregabalin, with the aim to investigate if they could be used in combination [44]. Using electrophysiological single-unit recordings taken from spinal wide dynamic range neurons, DNIC were assessed as a marker of descending controls in a model of OA. The question asked was whether tapentadol or pregabalin, both alone and in combination, inhibit neuronal responses and restore DNIC, quantified as a reduction in neuronal firing in the presence of noxious stimulus. Tapentadol restored DNIC-induced neuronal inhibition, while pregabalin inhibited preconditioned mechanically evoked neuronal responses but did not restore DNIC. This is perfectly in line with the actions of these drugs, with pregabalin acting at spinal levels on calcium channel modulation [45]. When a combination of the two drugs was given, DNIC expression returned and inhibition of spinal neuronal responses was seen. Thus, the combination therapy of tapentadol and 
pregabalin restored descending noradrenergic inhibitory tone and also inhibited nociceptive transmission at the spinal cord; however, this effect may be associated with increased risk of dizziness and sedation [46].

The pharmacological modulation of descending inhibition in humans has been studied by Niesters et al. investigating the influence of tapentadol on CPM (the human counterpart of DNIC), the descending inhibitory system that uses NA and subsequent activation of the $\alpha$-2-adrenoceptor and offset analgesia (a test in which a marked analgesia becomes apparent as a slight decrease in noxious heat stimulation is produced) [47]. In this study, 24 patients with diabetic polyneuropathy (DPN) were randomly assigned to either tapentadol prolonged release (PR) or placebo for 4 weeks. Before treatment, no patient showed CPM or offset analgesia. After 4 weeks of treatment, CPM was activated in the tapentadol PR group compared with placebo (24.2 vs 14.3\%); the relief of DPN pain was also greater in patients on tapentadol compared with those on placebo. On the basis of this evidence, the analgesic efficacy of tapentadol in patients with DPN appears to be dependent on the activation of descending inhibitory pain pathways. This translates perfectly to the preclinical data that also show a restoration of DNIC in neuropathic models. Most importantly, this preclinical study revealed that DNIC was an NA-mediated descending inhibition that was lost in a model of neuropathic pain. Thus, the ability of tapentadol to restore CPM is a perfect translation from the preclinical to clinical domain; moreover, these clinical data are in line with the NRI-mediated analgesic component of the drug. Accordingly, tapentadol has been described as "one key for two locks," since its synergistic action can represent an important option for the first-line treatment of patients who require both nociceptive and neuropathic pain relief (and therefore also for those with mixed pains), also given its favorable efficacy/side-effect ratio. In particular, the inhibition of NA reuptake is a key mechanism - and even predominates over opioid actions - in chronic pain states, and especially in neuropathic ones, thus making tapentadol markedly different to classical opioids. Moreover, reduced incidence of some typical opioid-induced side effects, compared with what reported in patients on equi-analgesic doses of classical opioids, further differentiate tapentadol from opioids.

\section{The newly introduced concept of $\mu$-load and the safety of tapentadol}

Importantly, the two mechanisms, MOR and NRI do not interact synergistically on the burden of adverse effects. This is an important issue since tolerability is as important as efficacy and the latter is the same as full $\mu$-opioids, but the side-effect profile is reduced in comparison.

In a recent study, the novel concept of $\mu$-load was introduced [2]. In short, a drug acting as an agonist at the MOR given systemically will act on all receptors in the body. Apart from analgesia, this will lead to nausea, vomiting, constipation, respiratory depression and other side effects that may also include dependence. This $\mu$-load should be reduced in drugs where there is no complete reliance on the MOR for analgesia. Indeed, tapentadol analgesic effect derives from the combined contribution of an opioid mechanism and NRI, the extent of which can vary for different pains as well as for adverse effects. Raffa and colleagues quantified both the MOR and the NRI component of the mechanism of action to both analgesia and to adverse effects in rodents [2]. The authors applied standard drug receptor theory and other approaches to a body of in vitro and in vivo data to estimate, in a number of different ways, the $\mu$-load of tapentadol (defined as the percentage contribution of the opioid component to the degree of adverse effects, such as respiratory depression and constipation, relative to a pure/classical $\mu$-opioid at equianalgesic doses) and related this to clinical data. The estimate was remarkably consistent and led to the conclusion that the $\mu$-load of tapentadol is $\leq 40 \%$ (relative to pure MOR agonists, such as morphine, oxycodone and fentanyl, which have by definition a $\mu$-load of $100 \%)$. This reduced $\mu$-load in clinical settings likely underlies the more favorable tolerability profile of tapentadol compared with strong classical opioids [48]. 
A comprehensive review has extensively discussed the tolerability profile of tapentadol on the bases of published studies and 'field-practice' post-marketing data, on a total of more than 22,000 patients [49]. Overall, 7185 subjects in clinical trials (57.5\%) and 777 patients included in 'field-practice' databases (7.3\%) showed tapentadol-related AEs. In all databases, $\mu$-receptor-dependent events, such as gastrointestinal, and CNS-related drug reactions were the most frequently reported, while noradrenergic-dependent reactions were negligible. No differences in the tolerability profile were disclosed between elderly and younger patients [49]. In addition, tapentadol appears to be better tolerated compared with opioids in studies evaluating specific adverse events, such as gastrointestinal events, hypertension, serotonin syndrome, endocrine toxicity, respiratory depression and, most importantly for young and adult patients, convulsions [48]. The large amount of data collected in clinical trials and in 'field-practice' studies strongly supports the premise of a very good tolerability profile of tapentadol. Importantly, safety data for this drug extend up to 4 years, a followup period considerably longer than for other opioids (up to 13 months in a recent Cochrane metaanalysis [50]).

Importantly, tapentadol shows minimal serotoninergic activity [51]. This pharmacodynamic profile may have a great relevance in chronic therapy, since the activation of serotoninergic pathways can produce proalgesic effects [45] and may also stimulate emesis over time [52]. Furthermore, there is a reduced risk of the serotonergic syndrome being produced through drug interactions between classical opioids and serotoninergic drugs, such as selective serotonin reuptake inhibitors (SSRIs). Studies in the US also suggest a limited risk of abuse with tapentadol $[53,54]$.

A favorable tolerability profile over a long-term period is a key determinant of treatment selection and patient compliance in clinical practice, and it is associated with improved QoL. We believe that the available safety data on tapentadol PRs support the use of this drug for the treatment of chronic painful conditions in which it has been shown analgesic efficacy.

\section{Clinical rationale for the use of tapentadol in chronic pain conditions}

Tapentadol has been extensively evaluated in chronic pain conditions, such as back pain, OA, cancer pain, and specifically in neuropathic pain. A detailed presentation of all clinical experiences with tapentadol in these settings goes beyond the aims of the present paper but can be found in a series of recently published reviews [15,55-57]. A paper highlighting the positive impact of tapentadol on QoL has also been recently published [58]. Here, we will focus on the characteristics of tapentadol which make it a suitable choice in the two pain models discussed above - that is, OA and low back pain.

\section{Osteoarthritis}

OA pain presents a wide heterogeneity of signs and symptoms [59], and in some cases, neuropathic mechanisms may also be involved, since structural changes of joint innervation, such as local loss and/or sprouting of nerve fibers are common in OA. In addition, central sensitization, reduction of descending inhibition, abnormal descending facilitation and cortical atrophy have been observed in OA patients with all of these mechanisms contributing to the transition to chronic pain and enhanced pain severity. Therefore, centrally acting analgesics become crucial to avoid the establishment of neuroplasticity phenomena leading to persistent pain [14]. As a consequence of the chronic pain, which is associated with functional limitations, patients may ultimately need to undergo knee or hip replacements. In most cases, the waiting period prior to surgery is characterized by persisting moderate-severe pain, with a limitation in the ability to perform daily activities and an increased risk of post-surgical pain. Control of pain is also important postoperatively, since pain is associated with longer hospitalization, modest compliance with the rehabilitation program, delayed return to daily activities and increased risk of postoperative complications [55]. On these bases, a molecule able to act both on the nociceptive and the neuropathic components of pain may be considered in the treatment of OA pain. Tapentadol meets these requirements, and therefore could be considered for 
the treatment of OA pain, although individual variability in response should be considered [60]. Remarkably, the reduced $\mu$-load of tapentadol helps reducing the burden of adverse effects compared with opioids, while retaining an at least equal efficacy.

These assumptions found some confirmations in clinical trials. In a randomized, double-blind study comparing tapentadol PR and oxycodone CR in 1030 patients with moderate-to-severe chronic OArelated knee pain, tapentadol $P R$, but not oxycodone $C R$, significantly reduced pain intensity from baseline to week 12 , and the proportion of patients with $\geq 50 \%$ improvement in pain intensity was higher with tapentadol PR ( $32.0 \%$ vs $17.3 \%$ with oxycodone CR and $24.3 \%$ with placebo [61]. In a subsequent open-label, phase IIIb study, tapentadol PR was effective in decreasing pain intensity and improving the Western Ontario and McMaster Universities OA index and QoL in patients with poor response to previous treatments or left untreated [62]. In another open-label study, patients with OA were randomly assigned to either tapentadol $(n=108)$ or etoricoxib $(n=110)$ for 12 weeks [63]. Steady improvement was seen in pain intensity and WOMAC scores in both groups, but a higher number of patients reported at least satisfactory response in the tapentadol group $(p=0.036)$. However, the above-mentioned findings were challenged by those reported in a double-blind, placebo or oxycodone CR-controlled trial, in which both tapentadol and oxycodone did not significantly reduce pain intensity vs placebo after 12 weeks of treatment [64], although the study did not demonstrate assay sensitivity and, therefore, the lack of efficacy of tapentadol cannot be fully interpreted.

\section{Chronic back pain}

On the other hand, CBP is a heterogeneous condition, characterized by both nociceptive and neuropathic pain [15]. In particular, nociceptive pain results from the activation of nociceptors as a response to tissue injury and biomechanical stress. The neuropathic component - which is often underestimated when selecting treatment - arises from injury of the nerve roots that innervate the spine and lower limbs, and pathological invasive innervation of the damaged lumbar discs [15]. Tapentadol PR has been proven to provide a strong analgesic effect in low back pain, due to its synergic MOR and NRI action, and a more favorable safety profile than opioids, also due to its reduced $\mu$-load $[14,15]$. In the pivotal trial of tapentadol in CBP, with a randomized, double-blind, placebo-controlled design, both tapentadol PR and oxycodone significantly reduced average pain intensity versus placebo at week 12 and throughout the maintenance period [65]. In another openlabel, uncontrolled, phase IIIb study in patients with poor tolerance to opioids, $80.9 \%$ of patients had $a \geq 50 \%$ reduced intensity of pain at week 6 [66]. A randomized, double-blind study comparing the effectiveness and tolerability of tapentadol PR monotherapy versus tapentadol PR/pregabalin combination therapy for severe CBP with a neuropathic component showed a similar efficacy of the two regimens [67]. Last, another randomized, controlled, open-label, phase 3IIlb/4IV study showed a superior change in pain intensity with tapentadol PR, compared with oxycodone/naloxone [68]. The efficacy of tapentadol in CBP is also supported by the results of a Cochrane review - although published in 2015, and including also studies on OA - which shows that tapentadol PR is associated with a reduction in pain intensity compared with placebo and oxycodone, and presents an improved safety profile compared with oxycodone [69]. Long-term safety and tolerability data on tapentadol PR corroborated its efficacy and safety [70]; however, long-term studies on tapentadol in CBP are still needed [15].

As discussed, there is preclinical evidence for combining pregabalin and tapentadol [44] and a case could be made for a rationale combination of a peripherally acting agent such as a NSAID or paracetamol with the MOR-NRI agents due to different mechanisms and sites of action. There is no basis for combination of tapentadol with opioids or TCA/SNRI drugs due to an overlap of mechanisms.

From the literature, it comes out that there is no established dosage of tapentadol for specific diseases. The common use of tapentadol is $100-250 \mathrm{mg}$ twice daily, depending of pain intensity. The 
dosage should be progressively increased on the basis of the effect on pain complained by the single patient [15].

The duration of therapy has no limits, given the low risk of adverse effects associated with tapentadol. A Cochrane review reports minimal adverse effects after 13 years of therapy [71]. Another paper confirms the reduced risk of side effects in comparison with opioids [48].

With regards to the combination of tapentadol with other analgesics, the paper by Otto et al. on low back pain [72] and that by Lockwood and Dickenson on osteoarthritis [44], both published in 2019, demonstrated the useful combination of tapentadol and pregabalin.

An open question remains on how and when to combine tapentadol with other analgesics, such a pregabalin, which target other molecular mechanisms involved in the establishment of chronic pain (i.e. glutamate release). A recent study conducted in LBP patients suggests that the combination therapy (tapentadol PR + pregabalin) exerts its best clinical efficacy in subjects characterized by severe sleep disturbances and little anxiety [72]. Moreover, a study by D'Amato et al. showed the efficacy of the combination of tapentadol and ketoprofen after hip arthroplasty [73]. The combination with other analgesics allows to reduce the dosage of tapentadol, although further dedicated studies on this treatment approach are required, since data obtained to date are not fully positive. Another possibility is the association of tapentadol with lidocaine patch, for localized neuropathic pain.

\section{Conclusion}

Solid evidence has highlighted the importance of central sensitization in the establishment to chronic pain [9]. Although there is still much to discover in this field, it appears that the noradrenergic system holds a major role in limiting central sensitization and the progression to chronic pain, by acting both on neurons and on, according to very recent results, on the glia. Therefore, pharmacological modulation of the noradrenergic system appears to be a well-grounded strategy for the control of chronic pain.

Tapentadol is characterized by a unique mechanism of action to date, since it acts both as a MOR agonist and as an inhibitor of NA reuptake. The synergistic interaction of these two MoA allows to achieve a strong analgesic effect by acting on both ascending and descending pathways [9].

Remarkably, the reduced $\mu$-load of tapentadol has two important consequences: first, it limits the risk of opioid-related adverse events, such as nausea/vomiting and respiratory depression, as well as the risk of dependence; second the NA component is predominant with consequent specific clinical efficacy on neuropathic and chronic pain [60].

These characteristics have led some authors to define tapentadol as an atypical opioid [52] and make it a suitable treatment for of chronic pain conditions characterized by both a nociceptive and a neuropathic component, such as OA or back pain. 


\section{References}

1. Caputi FF, Nicora M, Simeone R, et al. Tapentadol: an analgesic that differs from classic opioids due to its noradrenergic mechanism of action. Minerva Med. 2019;110(1):62-78.

2. Raffa RB, Elling C, Tzschentke TM. Does 'Strong analgesic' equal 'strong opioid'? Tapentadol and THE CONCEPT OF ' $\mu$-Load'. Adv Ther. 2018;35(10):1471-1484.

3. Dickenson AH, Kress HG. Tapentadol: a new option for the treatment of cancer and noncancer pains. J Pain Res. 2019;12:1509-1511.

4. Morlion B, Coluzzi F, Aldington D, et al. Pain chronification: what should a non-pain medicine specialist know? Curr Med Res Opin. 2018;34(7):1169-1178.

5. McCarberg B, Peppin J. Pain Pathways and nervous system plasticity: learning and memory in pain. Pain Med. 2019 Mar 13. pii: pnz017. doi: 10.1093/pm/pnz017.

6. Bourne S, Machado AG, Nagel SJ. Basic anatomy and physiology of pain pathways. Neurosurg Clin N Am. 2014;25(4):629-38.

7. Kuner R. Central mechanisms of pathological pain. Nat Med 2010;16(11):1258-66.

8. Ossipov MH, Morimura K, Porreca F. Descending pain modulation and chronification of pain. CurrOpin Support Palliat Care 2014;8(2):143-51.

9. Romualdi P, Grilli M, Canonico PL, et al. Pharmacological rationale for tapentadol therapy: a review of new evidence. J Pain Res. 2019;12:1513-1520.

10. Bannister K, Dickenson AH. What the brain tells the spinal cord. Pain. 2016;157(10):2148-51.

11. Bannister K, Dickenson AH. The plasticity of descending controls in pain: translational probing. J Physiol. 2017;595(13):4159-4166.

12. Tiwari V, Guan Y, Raja SN. Modulating the delicate glial-neuronal interactions in neuropathic pain: Promises and potential caveats. NeurosciBiobehav Rev 2014;45:19-27.

13. Caraci F, Sara Merlo, Filippo Drago, et al. Rescue of noradrenergic system as a novel pharmacological strategy in the treatment of chronic pain: focus on microglia activation. Front Pharmacol 2019;10:1024.

14. Coluzzi F, Fornasari D, Pergolizzi J, et al. From acute to chronic pain: tapentadol in the progressive stages of this disease entity. Eur Rev Med Pharmacol Sci. 2017;21(7):1672-1683.

15. Coluzzi F, Polati E, Freo U, et al. Tapentadol: an effective option for the treatment of back pain. J Pain Res. 2019;12:1521-1528.

16. Anand $P$, Dickenson $A$, Finco $G$, et al. Novel insights on the management of pain: highlights from the 'Science of Relief' meeting. Pain Manag. 2019 Sep 2. doi: 10.2217/pmt-2019-0031. [Epub ahead of print]

17. Thakur M, Dickenson AH, Baron R. Osteoarthritis pain: nociceptive or neuropathic? Nat Rev Rheumatol. 2014;10(6):374-80.

18. Jimenez-Andrade JM, Mantyh PW. Sensory and sympathetic nerve fibers undergo sprouting and neuroma formation in the painful arthritic joint of geriatric mice. Arthritis Res Ther. 2012;14:R101.

19. Ghilardi JR, Freeman KT, Jimenez-Andrade JM, et al. Neuroplasticity of sensory and sympathetic nerve fibers in a mouse model of a painful arthritic joint. Arthritis Rheum 2012;64:2223-2232.

20. Lockwood SM, Bannister K, Dickenson AH. An investigation into the noradrenergic and serotonergic contributions of diffuse noxious inhibitory controls in a monoiodoacetate model of osteoarthritis. J Neurophysiol. 2019;121(1):96-104.

21. Lluch E, Torres R, Nijs J, et al. Evidence for central sensitization in patients with osteoarthritis pain: a systematic literature review. Eur J Pain. 2014;18:1367-1375.

22. Dimitroulas T, Duarte RV, Behura A, et al. Neuropathic pain in osteoarthritis: a review of pathophysiological mechanisms and implications for treatment. Semin Arthritis Rheum 2014;44:145-154.

23. Fingleton C, Smart K, Moloney N, et al. Pain sensitization in people with knee osteoarthritis: a systematic review and meta-analysis. Osteoarthritis Cartilage 2015;23:1043-1056.

24. LluchGirbés $E$, Dueñas $L$, Barbero $M$, et al. Expanded distribution of pain as a sign of central sensitization in individuals with symptomatic knee osteoarthritis. Phys Ther. 2016;96:1196-1207. 
25. Steen Pettersen P, Neogi T, Magnusson K, et al. Peripheral and central sensitization of pain in individuals with hand osteoarthritis and associations with self-reported pain severity. Arthritis Rheumatol. 2019;71(7):1070-1077.

26. Carlesso LC, Segal NA, Frey-Law L, et al. Pain susceptibility phenotypes in those free of knee pain with or at risk of knee osteoarthritis: The Multicenter Osteoarthritis study. Arthritis Rheumatol. 2019;71(4):542-54.

27. Soni $A$, Wanigasekera V, Mezue $M$, et al. Central sensitization in knee osteoarthritis: relating presurgical brainstem neuroimaging and PainDETECT-based patient stratification to arthroplasty outcome. Arthritis Rheumatol. 2019;71(4):550-560. doi: 10.1002/art.40749.

28. Belluzzi E, Stocco E, Pozzuoli A, et al. Contribution of infrapatellar fat pad and synovial membrane to knee osteoarthritis pain. Biomed Res Int. 2019;2019:6390182.

29. Apkarian AV, Sosa Y, Sonty S, et al. Chronic back pain is associated with decreased prefrontal and thalamic gray matter density. J Neurosci 2004;24:10410-10415.

30. Fritz HC, McAuley JH, Wittfeld K, et al. Chronic back pain is associated with decreased prefrontal and anterior insular gray matter: results from a population-based cohort study. J Pain 2016;17:111-8.

31. Seminowicz DA, Wideman TH, Naso L, et al. Effective treatment of chronic low back pain in humans reverses abnormal brain anatomy and function. J Neurosci. 2011;31:7540-7550.

32. Arendt-Nielsen L, Morlion B, Perrot S, et al. Assessment and manifestation of central sensitisation across different chronic pain conditions. Eur J Pain. 2018;22(2):216-241.

33. Cohen E, Lee YC. A mechanism-based approach to the management of osteoarthritis pain. CurrOsteoporos Rep 2015;13:399-406.

34. Rojo ML, Rodríguez-Gaztelumendi A, Pazos Á, et al. Differential adaptive changes on serotonin and noradrenaline transporters in a rat model of peripheral neuropathic pain. NeurosciLett 2012;515:181-186.

35. Pertovaara A. The noradrenergic pain regulation system: a potential target for pain therapy. Eur J Pharmacol 2013;716:2-7.

36. Uchida $\mathrm{H}, \mathrm{Ma}$ L, Ueda $\mathrm{H}$. Epigenetic gene silencing underlies $\mathrm{C}-$ fiber dysfunctions in neuropathic pain. J Neurosci 2010;30:4806-4814.

37. Kress HG. Tapentadol and its two mechanisms of action: is there a new pharmacological class of centrally-acting analgesics on the horizon? Eur J Pain. 2010;14(8):781-3.

38. Tzschentke TM, Christoph T, Kögel BY. The mu-opioid receptor agonist/noradrenaline reuptake inhibition (MOR-NRI) concept in analgesia: the case of tapentadol. CNS Drugs. 2014;28(4):319-29.

39. Meske DS, Xie JY, Oyarzo J, et al. Opioid and noradrenergic contributions of tapentadol in experimental neuropathic pain. Neurosci Lett. 2014;562:91-6.

40. Bee LA, Bannister K, Rahman W, et al. Mu-opioid and noradrenergic $\alpha(2)$-adrenoceptor contributions to the effects of tapentadol on spinal electrophysiological measures of nociception in nerve-injured rats. Pain. 2011;152(1):131-9.

41. Torres-Sanchez S, Alba-Delgado C, Llorca-Torralba M, et al. Effect of tapentadol on neurons in the locus coeruleus. Neuropharmacology. 2013;72:250-8.

42. Falk $S$, Patel $R$, Heegaard $A$, et al. Spinal neuronal correlates of tapentadol analgesia in cancer pain: a back-translational approach. Eur J Pain. 2015;19(2):152-8.

43. Franchi S, Amodeo G, Gandolla M, et al. Effect of tapentadol on splenic cytokine production in mice. AnesthAnalg. 2017;124(3):986-995.

44. Lockwood SM, Dickenson AH. A combination pharmacotherapy of tapentadol and pregabalin to tackle centrally driven osteoarthritis pain. Eur J Pain. 2019;23(6):1185-1195.

45. Patel R, Dickenson AH. Modality selective roles of pro-nociceptive spinal 5-HT2A and 5-HT3 receptors in normal and neuropathic states. Neuropharmacology. 2018;143:29-37.

46. Baron R, Kern U, Müller M, Dubois C, Falke D, Steigerwald I. Effectiveness and tolerability of a moderate dose of tapentadol prolonged release for managing severe, chronic low back pain with a neuropathic component: an open-label continuation arm of a randomized phase $3 \mathrm{~b}$ study. Pain Pract. 2015;15(5):471-86. 
47. Niesters $\mathrm{M}$, Proto PL, Aarts $\mathrm{L}$, et al. Tapentadol potentiates descending pain inhibition in chronic pain patients with diabetic polyneuropathy. Br J Anaesth. 2014;113(1):148-56.

48. Polati E, Canonico PL, Schweiger V, et al. Tapentadol: an overview of the safety profile. J Pain Res. 2019;12:1569-1576.

49. Stollenwerk A, Sohns M, Heisig F, Elling C, von Zabern D. Review of post-marketing safety data on tapentadol, a centrally acting analgesic. Adv Ther. 2018;35(1):12-30. doi: 10.1007/s12325-0170654-0.

50. Els C, Jackson TD, Hagtvedt R, et al. High-dose opioids for chronic non-cancer pain: an overview of Cochrane Reviews. Cochrane Database Syst Rev. 2017;10:CD012299.

51. Thakur D, Dickerson S, Kumar Bhutani M, et al. Impact of prolonged-release oxycodone/naloxone on outcomes affecting patients' daily functioning in comparison with extended-release tapentadol: a systematic review. Clin Ther. 2015;37(1):212-24.

52. Pergolizzi JV Jr, LeQuang JA, Taylor R Jr, et al. Designing safer analgesics: a focus on $\mu$-opioid receptor pathways. Expert Opin Drug Discov. 2018;13(10):965-972.

53. Dart RC, Cicero TJ, Surratt HL, Rosenblum A, Bartelson BB, Adams EH. Assessment of the abuse of tapentadol immediate release: the first 24 months. J Opioid Manag. 2012;8(6):395-402.

54. Butler SF, McNaughton EC, Black RA. Tapentadol abuse potential: a postmarketing evaluation using a sample of individuals evaluated for substance abuse treatment. Pain Med. 2015;16(1):119-30.

55. Rinonapoli G, Coaccioli S, Panella L. Tapentadol in the treatment of osteoarthritis: pharmacological rationale and clinical evidence. J Pain Res. 2019;12:1529-1536.

56. Kress HG, Coluzzi F. Tapentadol in the management of cancer pain: current evidence and future perspectives. J Pain Res. 2019;12:1553-1560.

57. Freo U, Romualdi P, Kress HG. Tapentadol for neuropathic pain: a review of clinical studies. J Pain Res. 2019;12:1537-1551.

58. Panella L, Rinonapoli G, Coaccioli S. Where should analgesia lead to? Quality of life and functional recovery with tapentadol. J Pain Res. 2019;12:1561-1567.

59. Dell'Isola A, Steultjens M. Classification of patients with knee osteoarthritis in clinical phenotypes: Data from the osteoarthritis initiative. PLoS One. 2018;13(1):e0191045.

60. Langford RM, Knaggs R, Farquhar-Smith $\mathrm{P}$, et al. Is tapentadol different from classical opioids? A review of the evidence. Br J Pain. 2016;10(4):217-221.

61. Afilalo M, Etropolski MS, Kuperwasser B, et al. Efficacy and safety of Tapentadol extended release compared with oxycodone controlled release for the management of moderate to severe chronic pain related to osteoarthritis of the knee: a randomized, double-blind, placebo- and active-controlled phase III study. Clin Drug Investig. 2010;30(8):489-505.

62. Steigerwald I, Schenk M, Lahne U, Gebuhr P, Falke D, Hoggart B. Effectiveness and tolerability of tapentadol prolonged release compared with prior opioid therapy for the management of severe, chronic osteoarthritis pain. Clin Drug Investig. 2013;33(9):607-19.

63. Banerjee M, Mondal S, Sarkar R, Mondal H, Bhattacharya K. Comparative study of efficacy and safety of tapentadol versus etoricoxib in mild to moderate grades of chronic osteorthritis of knee. Indian Journal of Rheumatology 2016;11(1):21-25.

64. Serrie A, Lange B, Steup A. Tapentadol prolonged-release for moderate-to-severe chronic osteoarthritis knee pain: a double-blind, randomized, placebo- and oxycodone controlled release-controlled study. Curr Med Res Opin. 2017;33(8):1423-1432. doi: 10.1080/03007995.2017.1335189.

65. Buynak R, Shapiro DY, Okamoto A, et al. Efficacy and safety of tapentadol extended release for the management of chronic low back pain: results of a prospective, randomized, double-blind, placebo- and active-controlled Phase III study. Expert Opin Pharmacother. 2010;11(11):1787-804. doi: 10.1517/14656566.2010.497720.

66. Gálvez R, Schäfer M, Hans G, Falke D, Steigerwald I. Tapentadol prolonged release versus strong opioids for severe, chronic low back pain: results of an open-label, phase $3 b$ study. Adv Ther. 2013;30(3):229-59. 
67. Baron R, Martin-Mola E, Müller M, Dubois C, Falke D, Steigerwald I. Effectiveness and safety of tapentadol prolonged release (PR) versus a combination of tapentadol PR and pregabalin for the management of severe, chronic low back pain with a neuropathic component: a randomized, double-blind, phase 3b study. Pain Pract. 2015;15(5):455-70.

68. Baron R, Likar R, Martin-Mola E, et al. Effectiveness of tapentadol prolonged release (PR) compared with oxycodone/naloxone pr for the management of severe chronic low back pain with a neuropathic component: a randomized, controlled, open-label, phase $3 b / 4$ study. Pain Pract. 2016;16(5):580-99.

69. Santos J, Alarcão J, Fareleira F, et al. Tapentadol for chronic musculoskeletal pain in adults. Cochrane Database Syst Rev. 2015;(5):CD009923.

70. Buynak R, Rappaport SA, Rod K, et al. Long-term safety and efficacy of tapentadol extended release following up to 2 years of treatment in patients with moderate to severe, chronic pain: results of an open-label extension trial. Clin Ther. 2015;37(11):2420-38.

71. Els C, Jackson TD, Kunyk D, et al. Adverse events associated with medium- and long-term use of opioids for chronic non-cancer pain: an overview of Cochrane Reviews. Cochrane Database Syst Rev. 2017;10:CD012509.

72. Otto JC, Forstenpointner J, Sachau J, et al. A novel algorithm to identify predictors of treatment response: tapentadol monotherapy or tapentadol/pregabalin combination therapy in chronic low back pain? Front Neurol. 2019;10:979. doi: 10.3389/fneur.2019.00979.

73. D'Amato T, Martorelli F, Fenocchio G, et al. Tapentadol vs oxycodone/naloxone in the management of pain after total hip arthroplasty in the fast track setting: an observational study. J Exp Orthop. 2019;6(1):36. doi: 10.1186/s40634-019-0204-6. 


\section{Figure Legend}
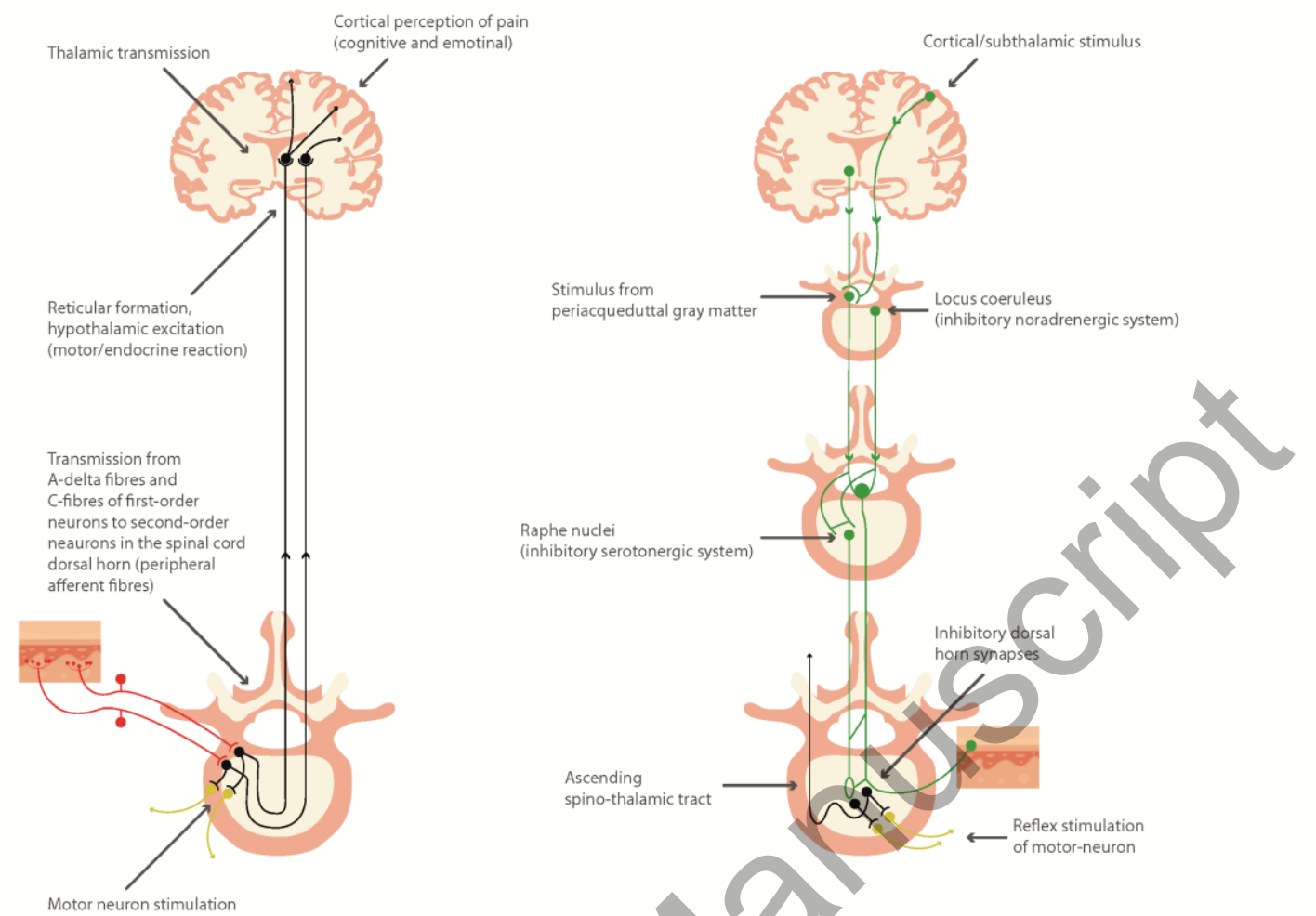

Figure 1. Ascending and descending pathways involved in pain transmission and modulation. For the descending pathways, nociceptive neurons located in the dorsal horn of the spinal cord project their axons to the thalamic neurons, forming the spinothalamic pathway and also other nociceptive ascending pathways. 
Table 1. Main types of pain and their definitions.

\begin{tabular}{|c|c|}
\hline Type of pain & Definition \\
\hline \multicolumn{2}{|c|}{ Location of the stimulus } \\
\hline Somatic & Pain experienced in the skin, muscles, bones, and joints \\
\hline Visceral & Pain experienced diffusely in the thoracic or abdominal cavities \\
\hline Mixed & A combination of the above \\
\hline \multicolumn{2}{|l|}{ Etiology } \\
\hline Nociceptive & $\begin{array}{l}\text { Pain due to nociceptor activation by high-threshold stimuli and their } \\
\text { transmission to the CNS }\end{array}$ \\
\hline Neuropathic & Pain induced by CNS or peripheral nervous system diseases or lesions \\
\hline Nociplastic & Pain without an obvious periphe \\
\hline Mixed & Pain including the previous co \\
\hline \multicolumn{2}{|l|}{ Duration } \\
\hline Acute & $\begin{array}{l}\text { Normally localized } \\
\text { Can persist up to a few days, diminishing with healing. } \\
\text { Usually caused by a trauma, infectious disease or surgical procedure. } \\
\text { Many effective treatments are available for the control of acute pain in the } \\
\text { majority of cases. }\end{array}$ \\
\hline Chronic & $\begin{array}{l}\text { Persists beyond the healing process } \\
\text { Caused by the persistence of harmful stimulus and/or by self-maintenance } \\
\text { phenomena which maintain the stimulation when the primary cause is } \\
\text { removed, leading to secondary hyperalgesia and central sensitization } \\
\text { Can refer to pain that accompanies chronic diseases } \\
\text { Difficult to treat, requires multidisciplinary treatment interventions }\end{array}$ \\
\hline
\end{tabular}

